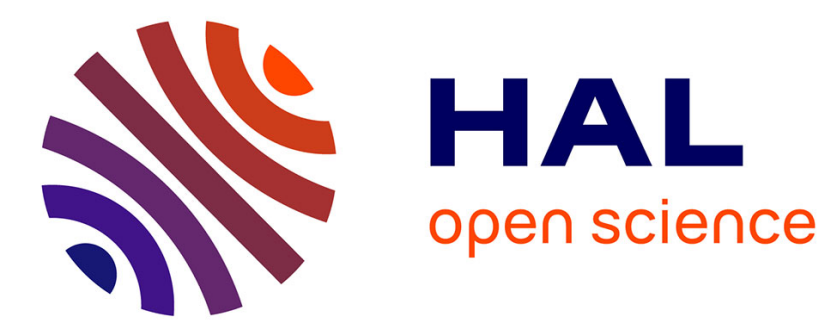

\title{
A Gramian-based observer with uniform convergence rate for delayed measurements
}

Juan G Rueda-Escobedo, Rosane Ushirobira, Denis Efimov, Jaime Moreno

\section{To cite this version:}

Juan G Rueda-Escobedo, Rosane Ushirobira, Denis Efimov, Jaime Moreno. A Gramian-based observer with uniform convergence rate for delayed measurements. ECC 2018 - 16th annual European Control Conference, Jun 2018, Limassol, Cyprus. hal-01895389

\section{HAL Id: hal-01895389 \\ https://hal.inria.fr/hal-01895389}

Submitted on 15 Oct 2018

HAL is a multi-disciplinary open access archive for the deposit and dissemination of scientific research documents, whether they are published or not. The documents may come from teaching and research institutions in France or abroad, or from public or private research centers.
L'archive ouverte pluridisciplinaire HAL, est destinée au dépôt et à la diffusion de documents scientifiques de niveau recherche, publiés ou non, émanant des établissements d'enseignement et de recherche français ou étrangers, des laboratoires publics ou privés. 


\title{
A Gramian-based observer with uniform convergence rate for delayed measurements
}

\author{
Juan G. Rueda-Escobedo, Rosane Ushirobira, \\ Denis Efimov, Jaime A. Moreno
}

\begin{abstract}
The problem of designing an observer for a linear time-invariant system with delayed measurements of the state is revisited in this paper. The delay is assumed to be time-varying with finite unknown lower and upper bounds. A Gramian-based observer is proposed with a fixed-time convergence rate to a ball. The efficiency of the obtained solution is illustrated by a numerical comparison with linear observers (one of which is tuned under the assumption that a nominal value of delay is available).
\end{abstract}

\section{INTRODUCTION}

State estimation is a well-studied problem in the theory of control systems and related domains [1], [2], [3], [4]. There are many estimation algorithms proposed for linear models [5], as well as numerous adaptations of these approaches to nonlinear cases [1]. Frequently, for an observer design some canonical forms are used in both scenarios, linear and nonlinear. The main issues that have to be analysed when designing an observer, include estimation error convergence rate [6], [7], robustness with respect to disturbances and measurement noises [8], [9], [10], overshooting and picking phenomenon of the errors [11], complexity of tuning, etc.

The problem becomes much more complicated if the output is available for measurements with delays, constant or time-varying, known or uncertain [12]. The complexity comes from difficulties in the stability and performance analysis for time-delay systems [13]. The reasons are originated by the fact that with time-delay, the system dynamics become infinite dimensional, and to find a Lyapunov-Krasovskii functional, to check stability or optimize performance, is a tricky problem even in the linear case [13]. In addition, the stability of a system independently in delays or with an augmented convergence rate (e.g. finite-time) is a complex and, frequently, nonlinear issue [14], while delay-dependent stability conditions are hardly applicable in practice since they need some evaluation of the admissible delay values and the rate of delay variation, which can be unavailable.

In the present work, we propose a direct application of the observer structure introduced in [15], [16] to the problem of observer design for a stable linear time-invariant plant with delayed measurements. In the nominal case, i.e. in the

J.G. Rueda-Escobedo and J.A. Moreno are with Eléctrica y Computación, Instituto de Ingeneiría, Universidad Nacional Autónoma de México, 04510 Mexico City, Mexico, JRuedaE@iingen.com; JMorenoPQi.unam.mx.

R. Ushirobira and D. Efimov are with Inria, Non-A team, Parc Scientifique de la Haute Borne, 40 avenue Halley, 59650 Villeneuve d'Ascq, France, Rosane.Ushirobira@inria.fr, Denis.Efimoveinria.fr. absence of delay or any other disturbances, this structure provides finite [16] and fixed-time [15] rates of convergence. In this work, it is assumed that the delay is time-varying and bounded, without restrictions on the speed of variation of the delay value. The upper and lower bounds for the delay are also assumed to be unknown, and not needed for the design. Under these hypothesis, an estimator is proposed, which use Gramian information calculated online demonstrating a uniform (fixed-time) rate of convergence of the estimation error to a ball (whose diameter is proportional to the uncertain delay). Despite the fact that the problem of designing fixed-time and finite-time converging observers became more popular recently [17], [18], [19], [20], [21], [22], [23], their advantages for observation in time-delay systems are not fully investigated yet.

The paper outline is as follows. The problem statement is given in Section II. Some preliminary results are discussed in Section III. The observer equations are introduced in Section IV. The analysis of the properties of the estimation error is carried out in Section V. The results of numerical experiments and a comparison with a linear observer are shown in Section VI. The final remarks and discussion are collected in Section VII.

Notation: The set of real numbers is denoted as $\mathbb{R}$. For a symmetric matrix $A \in \mathbb{R}^{n \times n}, \lambda_{M}(A)$ and $\lambda_{m}(A)$ denote the maximum and the minimum eigenvalues of $A$. For $s \in$ $\mathbb{R}$ its absolute value is denoted as $|s|$, while for a vector $s \in \mathbb{R}^{n}$ its $p^{\text {th }}$ norm is denoted by $\|s\|_{p}$ for any $p \geq 1$, and $\|s\|_{2}=\|s\|$; in addition, $\|s\|_{p} \leq\|s\| \leq n^{\frac{1}{2}-\frac{1}{p}}\|s\|_{p}$ for $p \geq 2$ and any $s \in \mathbb{R}^{n}$. The corresponding matrix norm of a matrix $A \in \mathbb{R}^{n \times n}$ is denoted by $\|A\|_{p}$, and $\|A\|_{2}=$ $\sqrt{\lambda_{M}\left(A^{\top} A\right)}$ in particular. For a scalar argument $s \in \mathbb{R}$ and $p \geq 0$, we use the notation $\lceil s\rfloor^{p}=|s|^{p} \operatorname{sign}(s)$, and it is applied elementwise in the case of a vector argument $s \in \mathbb{R}^{n}:\lceil s\rfloor^{p}=\left[\left|s_{1}\right|^{p} \operatorname{sign}\left(s_{1}\right) \cdots\left|s_{n}\right|^{p} \operatorname{sign}\left(s_{n}\right)\right]^{\top}$.

\section{Motivation AND PROBLEM STATEMENT}

Let us consider a linear time invariant system with a timevarying delay $\tau(t)$ in the output:

$$
\begin{aligned}
& \dot{x}(t)=A x(t)+B u(t), \\
& y(t)=C x(t-\tau(t)),
\end{aligned}
$$

where $x(t) \in \mathbb{R}^{n}$ and $y(t) \in \mathbb{R}^{p}$ are the state and the output vectors, respectively. In order to design a state feedback controller for (1), one must reconstruct the current state vector value $x(t)$ from the delayed output $y(t)$. To this end, introduce a conventional condition to design an observer: 
Assumption 1. The pair $(C, A)$ is observable.

Denote by $\hat{x}(t)$ an estimate of $x(t)$. The classical way to handle the problem is to use a copy of the system model with an injection of the output error delayed by $\tau(t)$ :

$$
\dot{\hat{x}}(t)=A \hat{x}(t)+B u(t)-L(C \hat{x}(t-\tau(t))-y(t)),
$$

which requires the value of the delay $\tau(t)$ to be known. Defining the estimation error as $e(t)=\hat{x}(t)-x(t)$, its dynamics results in

$$
\dot{e}(t)=A e(t)-L C e(t-\tau(t)),
$$

which correspond to a time-delay linear system. The stability of the previous system can be related to the matrix $A-L C$ being Hurwitz, and the feasibility of an algebraic Riccati inequality for a constant delay $\tau$ [24, Chap. 2]. Although the feasibility of such an inequality can be efficiently tested for a given $L$, using it for designing the gain may be difficult. For an arbitrary large $\tau$ it may not be possible to find a suitable $L$, then delay-dependent stability conditions and gains $L$ come to the focus. This problem can be avoided if the estimation is done by small steps as in [25] for constant delay, or as in [26] for time-varying ones, but the number of observers to be implemented may increase drastically. In order to use (2) for constant delay, one must know the length of the delay $a$ priori and store past data from the observer. If the delay is time-varying, the restrictions to be met by $A-L C$ becomes harder, and the delay, frequently, has to be known at each time instant.

Another solution is to apply the undelayed observer state in the output injection:

$$
\dot{\hat{x}}(t)=A \hat{x}(t)+B u(t)-L(C \hat{x}(t)-y(t)),
$$

which skips the requirement on availability of the delay value, then

$$
\dot{e}(t)=(A-L C) e(t)+L(y(t)-C x(t)),
$$

and the boundedness of the estimation error $e(t)$ follows the same property of the term $y(t)-C x(t)$ (the matrix $A-L C$ can be taken Hurwitz by a proper selection of $L$ due to Assumption 1). In such a case the delay can be assumed to be uncertain, time-varying and bounded. The issue of this scheme is that increasing the convergence rate (by augmenting $L$ ) usually leads to a loss of precision (since the perturbation term is also proportional to $L$ ):

Lemma 1. Let $P_{L}=P_{L}^{\top}>0$ and $Q_{L}=Q_{L}^{\top}>0$ be solutions of the Lyapunov equation

$$
(A-L C)^{\top} P_{L}+P_{L}(A-L C)=-Q_{L}
$$

for the given $L$. Then in (5) the error $e(t)$ stays bounded for all $t \geq t_{0} \geq 0$ and

$$
\lim _{t \rightarrow+\infty}\|e(t)\| \leq\|L\| \frac{\sqrt{\lambda_{M}\left(Q_{L}\right) \lambda_{M}^{3}\left(P_{L}\right)}}{\lambda_{m}\left(Q_{L}\right)} \sup _{t \geq 0}\|y(t)-C x(t)\| .
$$

Therefore, the previous approaches realized by (2) and (4) present some disadvantages. The objective of this note is to present a methodology to design a state observer, which alleviates most of these problems having a bounded error (the error upper bound has to be proportional to the delay), under the following hypothesis:

Assumption 2. For all $t \geq t_{0} \geq 0$ : $0 \leq \tau(t) \leq \tau^{\star}<+\infty$.

These bounds for the delay are not restrictive comparing to the existing results in the literature.

Assumption 3. For all $t \geq t_{0} \geq 0$ :

$$
\|u(t)\|<+\infty,\|y(t)-C x(t)\|<+\infty .
$$

For example, the conditions of Assumption 3 are satisfied if the matrix $A$ is Hurwitz, or if the control $u(t)$ ensures boundedness of the system trajectories. Thus, the last condition may restrict (1) to be asymptotically stable. In such a case, one can use as an observer just a copy of the plant, without output injection and having an exponentially stable error dynamics. However, in this design there is no control over the convergence velocity, and one has to relay on the intrinsic properties of the system inscribed in $A$. In this work a more sophisticated design is proposed allowing the convergence rate of the estimation error to be adjusted, but due to the lack of knowledge about the delay, the price to pay is to have a steady-state error in the estimation.

\section{PReliminaries}

In this section some developments that are used for the observer design are introduced and discussed. The main topic is how to use a Gramian-like construction to get an implicit undelayed state estimation error, which can be injected in the observer to improve the convergence rate.

To explain our idea, consider again the system (1) with an output feedback gain $K \in \mathbb{R}^{n \times p}$ assuming that there is no delay $(\tau=0)$ :

$$
\dot{x}(t)=(A+K C) x(t)+B u(t)-K y(t) .
$$

Since the only difference between (1) and (6) consists in the output injection, then both systems are observable through the output $y(t)=C x(t)$ and in the absence of delay [27]. The reason for introduction of (6) is that now the matrix $K$ represents a degree of freedom in our hand, which we are going to exploit for the convergence speed adjustment and for a Gramian calculation below. The constructibility Gramian $M(t)$ of (1) can be obtained online by implementing the following matrix differential equation:

$$
\dot{M}(t)=-A^{\top} M(t)-M(t) A+C^{\top} C, M\left(t_{0}\right)=0 .
$$

The solution of the previous equation is unbounded if there is any stable mode in (1), since in the adjoint system $\dot{z}(t)=$ $-A^{\top} z(t)$ this mode becomes unstable. Now consider the analogous equation for the constructability Gramian $N(t)$ of the system (6):

$$
\begin{aligned}
\dot{N}(t) & =-(A+K C)^{\top} N(t)-N(t)(A+K C)+C^{\top} C, \\
N\left(t_{0}\right) & =0 .
\end{aligned}
$$


Since the pair $(C, A)$ is observable by Assumption 1, the pair $\left(-A^{\top}, C^{\top}\right)$ is controllable and one can chose $K$ to make $-(A+K C)^{\top}$ Hurwitz, and the solutions of (7) are uniformly bounded. Furthermore, since the observability of (6) follows the same property of (1), then $N(t)$ becomes invertible for all $t>t_{0}$.

Remark 1. The online computation of $N(t)$ can be avoided using its final value

$$
N_{\infty}=\int_{0}^{+\infty} e^{-(A+K C)^{\top} t} C^{\top} C e^{-(A+K C) t} d t
$$

derived from the algebraic Lyapunov equation (7) for $\dot{N}(t)=$ 0 :

$$
(A+K C)^{\top} N_{\infty}+N_{\infty}(A+K C)=C^{\top} C .
$$

The error introduced by such a substitution disappears exponentially fast, leaving the result unaltered.

Now consider the relation $\psi_{N}(t)=N(t) x(t)$, where the variable $\psi_{N}(t)$ has the following dynamics:

$$
\begin{aligned}
\dot{\psi}_{N}(t)= & -(A+K C)^{\top} \psi_{N}(t)+\left(C^{\top}-N(t) K\right) C x(t) \\
& +N(t) B u(t) ; \quad \psi_{N}\left(t_{0}\right)=0,
\end{aligned}
$$

which is obtained by taking the time derivative of $\psi_{N}(t)=$ $N(t) x(t)$ and substituting the relation itself. Using $N(t)$ and $\psi_{N}(t)$, which are both variables available for measurements by their construction, a state estimate can be recovered as

$$
\hat{x}(t)=N^{-1}(t) \psi_{N}(t)
$$

Unfortunately, such a direct inversion has some drawbacks. First, $N(t)$ can be ill conditioned for $t$ close to $t_{0}$; second, from the time derivative of $\hat{x}(t)$ it is clear that there is no control over the convergence rate; third, such an operation can be computationally costly. Instead, the variables $N(t)$ and $\psi_{N}(t)$ can be used to create a correction term as

$$
N(t) \hat{x}(t)-\psi_{N}(t)=N(t)(\hat{x}(t)-x(t)) .
$$

Since $N(t)$ becomes invertible for all $t>t_{0}$, the correction term vanishes only if the estimation error is zero.

Until now, it has been assumed that the output is available for measurements without delay. Since this is not the case in our study, let us consider (8), but with the delayed output:

$$
\begin{aligned}
\dot{\psi}(t)= & -(A+K C)^{\top} \psi(t)+\left(C^{\top}-N(t) K\right) y(t) \\
& +N(t) B u(t) ; \quad \psi\left(t_{0}\right)=0,
\end{aligned}
$$

Denote by $\Delta_{y}(t)=y(t)-C x(t)$ and by $e_{\psi}(t)=\psi(t)-$ $\psi_{N}(t)$ the error introduced by the delay, its dynamics is given by

$$
\begin{aligned}
\dot{e}_{\psi}(t) & =-(A+K C)^{\top} e_{\psi}(t)+\left(C^{\top}-N(t) K\right)(y(t)-C x(t)) \\
& =-(A+K C)^{\top} e_{\psi}(t)+\left(C^{\top}-N(t) K\right) \Delta_{y}(t) .
\end{aligned}
$$

Since $-(A+K C)^{\top}$ is a stable matrix by design, then $N(t)$ by construction is bounded and converges to a constant, and the error $e_{\psi}(t)$ will remain bounded if the difference $\| y(t)-$ $C x(t) \|$ is uniformly bounded (Assumption 3). Furthermore, one has a certain degree of control on the size of the ultimate bound of $e_{\psi}(t)$ by means of $K$ :
Lemma 2. Let $P_{K}=P_{K}^{\top}>0$ and $Q_{K}=Q_{K}^{\top}>0$ be solutions of the Lyapunov equation

$$
(A+K C)^{\top} P_{K}+P_{K}(A+K C)=Q_{K}
$$

for a given $K$. Then in (10) the error $e_{\psi}(t)$ stays bounded for all $t \geq t_{0} \geq 0$ and

$\lim _{t \rightarrow+\infty}\left\|e_{\psi}(t)\right\| \leq\left\|C^{\top}-N_{\infty} K\right\|_{2} \frac{\lambda_{M}^{0.5}\left(Q_{K}\right) \lambda_{M}^{1.5}\left(P_{K}\right)}{\lambda_{m}^{0.5}\left(Q_{K}\right)} \sup _{t \geq 0}\left\|\Delta_{y}(t)\right\|$.

Now, we are in position to introduce the structure of the observer and its properties in the following section.

\section{MAIN RESUlT}

In this section we will use the relation $\psi(t)=N(t) x(t)+$ $e_{\psi}(t)$ developed in the previous section to correct the estimate of the internal state. Let $\hat{x}(t)$ be the estimate of $x(t)$. The term $N(t) \hat{x}(t)-\psi(t)$ is indeed a measure of the error perturbed by $e_{\psi}(t)$ :

$$
N(t) \hat{x}(t)-\psi(t)=N(t)(\hat{x}(t)-x(t))-e_{\psi}(t) .
$$

Thus, this expression can be used in the observer instead of the classical output injection term $C \hat{x}(t-\tau(t))-y(t)$, which is responsible for introducing the delay in the equation. Additionally, a priori knowledge on the size of the delay is not needed to build the proposed term, and it is proportional to the whole state and not only output variables. Now, consider a copy of the plant plus two correction terms represented in the following observer equation:

$$
\begin{gathered}
\dot{\hat{x}}(t)=A \hat{x}(t)+B u(t)-P^{-1} N(t)\left(k_{1}(N(t) \hat{x}(t)-\psi(t))\right. \\
\left.+k_{2}\lceil N(t) \hat{x}(t)-\psi(t)\rfloor^{p}\right)
\end{gathered}
$$

where the signals $N(t)$ and $\psi(t)$ are computed as in (7) and (9), respectively; $k_{1}>0, k_{2}>0$ and $p>1$ are tuning parameters to be chosen, and an invertible matrix $P$ is a solution the following Lyapunov algebraic equation:

Assumption 4. There exist $P=P^{\top}>0$ and $Q=Q^{\top} \geq 0$ such that $P A+A^{\top} P=-Q$.

We are in position to formulate the main result of the paper.

Theorem 1. Let assumptions 1-4 be satisfied for (1), and consider the observer (7), (9), (12) for $K \in \mathbb{R}^{n \times p}$ such that $-(A+K C)^{\top}$ is Hurwitz, with $k_{1}>0, k_{2}>0$ and $p>1$. Under these conditions, the estimation error $e(t)=$ $\hat{x}(t)-x(t)$ is ultimately bounded, admitting the following asymptotic estimate:

$$
\begin{aligned}
\sup _{t \geq T_{\eta}}\|e(t)\| \leq \eta \gamma \| C^{\top}- & N_{\infty} K \|_{2} \frac{\sqrt{\lambda_{M}\left(Q_{K}\right) \lambda_{M}^{3}\left(P_{K}\right)}}{\lambda_{m}\left(Q_{K}\right)} \\
& \times \sup _{t \geq 0}\|y(t)-C x(t)\|,
\end{aligned}
$$

with

$$
\gamma=\max \left\{\frac{1}{\sqrt{k_{1}^{-1} \lambda_{m}(Q)+\lambda_{m}^{2}\left(N_{\infty}\right)}}, \frac{\sqrt[p+1]{2 p^{p}\left[2^{p} p^{p+1}+2\right]}}{(p+1) \lambda_{m}\left(N_{\infty}\right)}\right\} .
$$


This bound is reached globally in a uniform time $T_{\eta}>0$ (independent on initial conditions) for any $\eta>1$.

The main feature of (12) consists in the gain $k_{2}>0$, which ensures entrance of all trajectories in the ball around the origin proportional to $\sup _{t>0}\|y(t)-C x(t)\|$ in a fixed time. In other words, the nonlinear term with exponent $p$ gives to the algorithm a very nice property. Usually, for any convergent algorithm, when the initial error grows, the time needed to reach any bounded region of zero goes to infinity. This does not happen with the algorithm (7), (9), (12). The uniformity with respect to the initial error means that the convergence time may increase, but there is a finite upper bound reached at infinity. To the best knowledge of the authors, this property is new for this class of problems. The gain of the linear term, $k_{1}$, controls the rate of convergence in the neighborhood of zero, whereas $k_{2}$ improve it far from this region.

Remark 2. The main advantage of the proposed approach is that the convergence rate can be assigned arbitrarily using $k_{1}$ and $k_{2}$, which do not influence the asymptotic precision of the observer, in contrast to the classical approach (2), where making the real par of the eigenvalues of $A-L C$ more negative might result in instability, or (4), where increasing of $L$ leads to the accuracy loss.

In the next section, the convergence analysis will be presented, together with the proof of Theorem 1 .

\section{ERROR ANALYSIS AND SKETCH OF PROOF OF THEOREM 1}

To study the convergence of the algorithm, and to show the properties given in the previous section, the dynamics of the estimation error $e(t)=\hat{x}(t)-x(t)$ is going to be analyzed:

$$
\begin{aligned}
\dot{e}(t)=A e(t)- & k_{1} P^{-1} N(t)\left(N(t) e(t)+e_{\psi}(t)\right) \\
& -k_{2} P^{-1} N(t)\left\lceil N(t) e(t)+e_{\psi}(t)\right\rfloor^{p} .
\end{aligned}
$$

To establish a convergence region, let us consider the positive definite function $V(e)=e^{\top} P e$. The derivative of $V$ along (13) is

$$
\begin{aligned}
\dot{V}(t)=- & e^{\top}(t) Q e(t)-2 k_{1} e^{\top}(t) N(t) N(t) e(t) \\
& -2 k_{2} e^{\top}(t) N(t)\left[N(t) e(t)+e_{\psi}(t)\right\rfloor^{p} \\
& -2 k_{1} e^{\top}(t) N(t) e_{\psi}(t) .
\end{aligned}
$$

The term inside semi-brackets can be split in two, one depending only on the observer error and other depending only on the error committed in the calculation of $\psi$. Specifically, the next inequality is going to be used:

$$
\nu^{\top}\lceil\nu+\delta\rfloor^{p} \geq 0.5\|\nu\|_{p+1}^{p+1}-\frac{2^{p} p^{2 p+1}+2 p^{p}}{(p+1)^{p+1}}\|\delta\|_{p+1}^{p+1},
$$

which is valid for any $\nu, \delta \in \mathbb{R}^{n}$ and $p>1$. Due to space limitations, the proof of (15) is omitted. Combining (15) and (14), one gets:

$$
\begin{aligned}
\dot{V}(t) \leq & -e^{\top}(t) Q e(t)-k_{1}\|N(t) e(t)\|^{2}-k_{2}\|N(t) e(t)\|_{p+1}^{p+1} \\
& +\frac{2 k_{2} p^{p}\left[2^{p} p^{p+1}+2\right]}{(p+1)^{p+1}}\left\|e_{\psi}(t)\right\|_{p+1}^{p+1}+k_{1}\left\|e_{\psi}(t)\right\|^{2} .
\end{aligned}
$$

Because the system is instantaneously observable, $N(t)$ is invertible for any $t>t_{0}$ (in the case of $N(t)$, its maximum eigenvalue can be obtained from its final value $N_{\infty}$ ), the last inequality can be transformed into (given that $\left.V^{\frac{1}{2}}(e) / \lambda_{M}^{\frac{1}{2}}(P) \leq\|e(t)\|\right)$

$$
\begin{aligned}
& \dot{V}(t) \leq- \frac{\lambda_{m}(Q)+k_{1} \lambda_{m}^{2}(N(t))}{\lambda_{M}(P)} V(t)-\frac{k_{2} \lambda_{m}^{p+1}(N(t))}{n^{\frac{1}{2}-\frac{1}{p+1}} \lambda_{M}^{\frac{p+1}{2}}(P)} V^{\frac{p+1}{2}}(t) \\
&+\frac{2 k_{2} p^{p}\left[2^{p} p^{p+1}+2\right]}{n^{\frac{1}{2}-\frac{1}{p+1}}(p+1)^{p+1}}\left\|e_{\psi}(t)\right\|^{p+1}+k_{1}\left\|e_{\psi}(t)\right\|^{2} .
\end{aligned}
$$

Since $(p+1) / 2>1$, one can conclude that the convergence of the system to a compact region containing the origin is uniform with respect to the initial value [28] (in other words, the time needed to reach the compact region accepts a constant upper bound which is independent from the initial value). In addition, $\dot{V}(t)<0$ provided that $\|e(t)\|>$ $\gamma\left\|e_{\psi}(t)\right\|$, with $\gamma$ as in Theorem 1. The required bound follows taking into account the result of Lemma 2.

\section{Simulation EXAMPLE}

In this section, the proposed observer is going to be designed for the system

$$
\begin{aligned}
\dot{x}(t) & =\left[\begin{array}{cc}
-0.5 & 2 \\
-1 & 0
\end{array}\right] x+\left[\begin{array}{l}
0 \\
1
\end{array}\right] u(t), \\
x(0) & =[30,-5]^{\top}, \\
y(t) & =\left[\begin{array}{ll}
1 & 0
\end{array}\right] x(t-\tau(t)),
\end{aligned}
$$

with input $u(t)=5 \sin (2 t)-3 \sin (3 t)$. A delay of $\tau(t)=$ $\alpha \sin (4 t)+0.35$ is introduced. This modeled a nominal delay of 0.35 perturbed by $\alpha$ which take values: $0.05,0.15,0.25$.

The proposed observer is compared with a delayed Luenberger observer (2) and the undelayed version (4). The first one is designed considering the nominal delay of 0.35 . The second one ignores the presence of the delay completely.

In the following, the parameters for each observer are presented:

- Proposed observer:

- $K=[7.5,-5]^{\top}$ such that the eigenvalues of $-(A+$ $K C)^{\top}$ are $\lambda_{1}=-3$ and $\lambda_{2}=-4$.

- The matrix $P$ was selected to meet the equation $P A+$ $A^{\top} P=-\mathbb{I}$, i.e.

$$
P=\left[\begin{array}{cc}
1.5 & -0.25 \\
-0.25 & 3.125
\end{array}\right]
$$

- The exponent $p$ was chosen as 2 . Both gains were set in $k_{i}=10$.

- Delayed Luenberger observer:

- $L=[1.3,-0.6]^{\top}$ with eigenvalues of $A-L C$ at $\lambda_{1}=$ -1 and $\lambda_{2}=-0.8$.

- The error dynamics satisfy [24, Theo. 2.2] for $\tau^{\star}=$ $0.35, \epsilon=3, \alpha=2$, and

$$
X=\left[\begin{array}{cc}
2.627 & 0.2818 \\
0.2818 & 0.556
\end{array}\right] .
$$

- Undelayed Luenberger observer: 
- $L=[1.3,-0.6]^{\top}$ with eigenvalues of $A-L C$ at $\lambda_{1}=$ -1 and $\lambda_{2}=-0.8$.

The initial conditions were set in zero. In the case of the delayed Luenberger observer, the initial function $\hat{x}=0$ for $t \leq t_{0}$ was chosen. The following plots show the norm of the estimation error $\|\hat{x}(t)-x(t)\|$ for the different values of $\alpha$ and for the different observers. Notice that the error

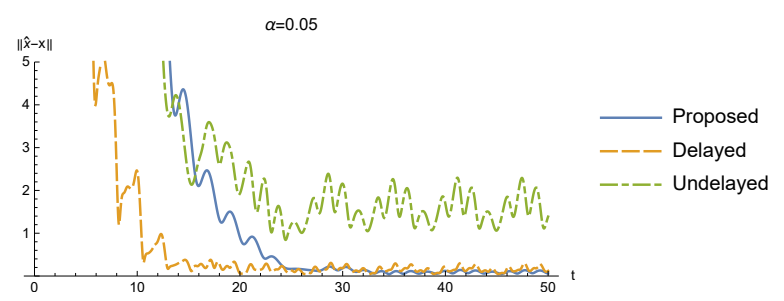

Fig. 1. Error behavior for $\tau(t)=0.05 \sin (4 t)+0.35$.

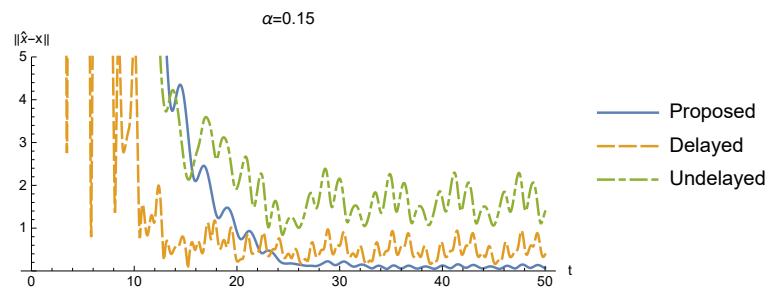

Fig. 2. Error behavior for $\tau(t)=0.15 \sin (4 t)+0.35$.

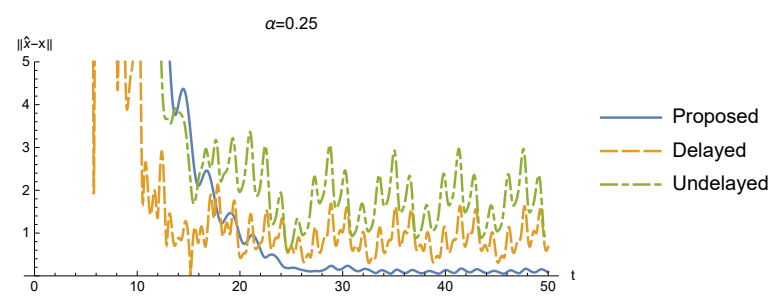

Fig. 3. Error behavior for $\tau(t)=0.25 \sin (4 t)+0.35$.

bound does not change consistently with $\alpha$ for the proposed observer and for the undelayed Luenberger observer. This happens because in these cases the error depends on the size of the difference $y(t)-C x(t)$ and not on the size of the delay itself. The delayed Luenberger observer can estimate correctly the internal state for the nominal delay, but in the perturbed case the error grows with $\alpha$.

Now, to illustrate the fixed-time convergence to a region, the initial condition for the proposed observer was increased so that it have values in the order of $10^{2}, 10^{4}, 10^{6}$, and $10^{8}$. $\alpha=0.25$ was set in this case. As can be seen in Figure 4, the convergence is accelerated as the initial error is increased. The final region is reached at almost the same time in all the cases. In contrast, attempting to increase the convergence rate by increasing the gain in the undelayed Luenberger observer results in a lager error. In the case of the delayed Luenberger observer, increasing the gain may result in instability.

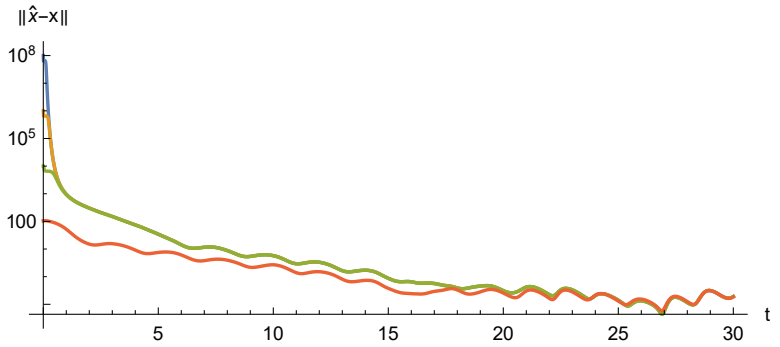

Fig. 4. Logarithmic plot to illustrate the fixed-time convergence.

\section{CONCLUSIONS}

In this note, a methodology to design observers for stable linear time-invariant systems with an unknown timevarying and uniformly bounded delay in the output was presented. To design the observer, no a priori knowledge of the delay is needed. In contrast, only convergence to a compact region of the estimation error is achieved, since the lack of knowledge about the delay complicates the convergence to zero. The design of the observer only involves the specification of a positive definite matrix, and requires to solve a set of Lyapunov algebraic equation. The boundedness of the estimation error is guaranteed for any finite delay, and for any observer gain. However, the size of the error bound increases while increasing the observer gain. The radius of the ball depends on the difference between the nominal output and the delayed (available) one, and not directly on the size of the delay and/or its derivative. The proposed new estimator is compared with the behavior of a standard Luenberger observer for delayed systems by means of numerical simulation. It was observed that the ultimate bound of the estimation error with the Luenberger observer increases drastically when the nominal value of the delay is perturbed, whereas for the proposed observer, designed without knowledge of the delay, it remains the same.

\section{ACKNOWLEDGMENTS}

This work is supported in part by: HoTSMoCE Inria associate team program, ANR Finite4SoS (ANR 15 CE23 0007); by the Government of Russian Federation (Grant 074U01); by the Ministry of Education and Science of Russian Federation (Project 14.Z50.31.0031); by the financial support from PAPIIT-UNAM (Programa de Apoyo a Proyectos de Investigación e Innovaciónn Tecnológica), project IN113617; Fondo de Colaboración II-FI UNAM, Project IISGBAS100-2015; CONACyT (Consejo Nacional de Ciencia y Tecnología), project 241171 and CONACyT CVU 491701.

\section{REFERENCES}

[1] G. Besançon, Ed., Nonlinear Observers and Applications, ser. Lecture Notes in Control and Information Sciences. Berlin: Springer-Verlag, 2007, vol. 363.

[2] A. Astolfi and L. Marconi, Eds., Analysis and Design of Nonlinear Control Systems. Berlin: Springer-Verlag, 2008.

[3] T. Meurer, K. Graichen, and E.-D. Gilles, Eds., Control and Observer Design for Nonlinear Finite and Infinite Dimensional Systems, ser. Lecture Notes in Control and Information Sciences. Berlin: SpringerVerlag, 2005, vol. 322. 
[4] J. L. Crassidis and J. L. Junkins, Optimal Estimation of Dynamic Systems, 2nd ed. CRC Press, 2012.

[5] D. G. Luenberger, Introduction to dynamic systems: theory, models, and applications. Wiley, 1979.

[6] W. Perruquetti, T. Floquet, and E. Moulay, "Finite-time observers: application to secure communication," IEEE Transactions on Automatic Control, vol. 53, no. 1, pp. 356-360, 2008.

[7] T. Raff and F. Allgöwer, "An observer that converges in finite time due to measurement-based state updates," $\{$ IFAC $\}$ Proceedings Volumes, vol. 41, no. 2, pp. 2693-2695, 2008, 17th \{IFAC $\}$ World Congress.

[8] L. K. Vasiljevic and H. K. Khalil, "Error bounds in differentiation of noisy signals by high-gain observers," Systems \& Control Letters, vol. 57, no. 10, pp. 856-862, 2008.

[9] J. H. Ahrens and H. K. Khalil, "High-gain observers in the presence of measurement noise: A switched-gain approach," Automatica, vol. 45, no. 4, pp. 936-943, 2009.

[10] R. G. Sanfelice and L. Praly, "On the performance of high-gain observers with gain adaptation under measurement noise," Automatica, vol. 47, no. 10, pp. 2165-2176, 2011.

[11] P. Kokotovic, "The joy of feedback: nonlinear and adaptive," IEEE Control Systems Magazine, vol. 12, no. 3, pp. 7-17, 1992.

[12] S.-I. Niculescu and K. Gu, Eds., Advances in Time-Delay Systems. Berlin: Springer-Verlag, 2004, vol. 38.

[13] E. Fridman, Introduction to Time-Delay Systems. Basel: Birkhäuser, 2014.

[14] D. Efimov, A. Polyakov, W. Perruquetti, and J. P. Richard, "Weighted homogeneity for time-delay systems: Finite-time and independent of delay stability," IEEE Transactions on Automatic Control, vol. 61, no. 1, pp. 210-215, Jan 2016

[15] J. G. Rueda-Escobedo, J. A. Moreno, and P. Oliva-Fonseca, "Fixedtime convergent unknown input observer for LTI systems," in XVII Latin American Conference in 2016 Automatic Control, Oct 2016, pp. 354-359. [Online]. Available: https://goo.gl/BHxzNu

[16] _ "Finite-time state estimation for LTI systems with a first-order sliding mode," in 2016 14th International Workshop on Variable Structure Systems (VSS), June 2016, pp. 194-199.

[17] V. Andrieu, L. Praly, and A. Astolfi, "Homogeneous Approximation, Recursive Observer Design, and Output Feedback," SIAM J. Control Optimization, vol. 47, no. 4, pp. 1814-1850, 2008.

[18] E. Cruz-Zavala, J. A. Moreno, and L. M. Fridman, "Uniform robust exact differentiator," IEEE Transactions on Automatic Control, vol. 56, no. 11, pp. 2727-2733, Nov 2011.

[19] E. Cruz-Zavala, J. A. Moreno, and L. Fridman, "Asymptotic stabilization in fixed time via sliding mode control," in Decision and Control (CDC), 2012 IEEE 51st Annual Conference on, Dec 2012, pp. 64606465.

[20] A. Polyakov, "Fixed-time stabilization of linear systems via sliding mode control," in Variable Structure Systems (VSS), 2012 12th International Workshop on, Jan 2012, pp. 1-6.

[21] H. Ríos and A. Teel, "A hybrid observer for uniform finite-time state estimation of linear systems," in In Proc. 55th IEEE Conference on Decision and Control (CDC), Las Vegas, 2016.

[22] F. Lopez-Ramirez, D. Efimov, A. Polyakov, and W. Perruquetti, "Fixed-time output stabilization of a chain of integrators," in Proc. 55th IEEE Conference on Decision and Control (CDC), Las Vegas, 2016.

[23] E. Cruz-Zavala and J. A. Moreno, "Lyapunov functions for continuous and discontinuous differentiators," IFAC-PapersOnLine, vol. 49, no. 18 , pp. $660-665,2016$.

[24] M. S. Mahmoud, Robust Control and Filtering for Time-Delay Systems (Automation and Control Engineering). CRC Press, 2000.

[25] G. Besançon, D. Georges, and Z. Benayache, "Asymptotic state prediction for continuous-time systems with delayed input and application to control," in 2007 European Control Conference (ECC), July 2007, pp. 1786-1791.

[26] V. Léchappé, E. Moulay, and F. Plestan, "Dynamic observationprediction for lti systems with a time-varying delay in the input," in 2016 IEEE 55th Conference on Decision and Control (CDC), Dec 2016, pp. 2302-2307.

[27] L. Zhang and Q. Zhang, "Observability conservation by output feedback and observability gramian bounds," Automatica, vol. 60, pp. 38 $-42,2015$.

[28] E. Bernuau, D. Efimov, W. Perruquetti, and A. Polyakov, "On homogeneity and its application in sliding mode control," Journal of the Franklin Institute, vol. 351, no. 4, pp. 1866 - 1901, 2014. 